\title{
CINCO ESPÉCIES DE LECITHODENDRIIDAE (TREMATODA) EN MURCIELAGOS DE COLOMBIA, INCLUYENDO TRES NUEVOS GENEROS
}

\author{
Imelda Velez ${ }^{1}$ \\ Vernon E. Thatcher ${ }^{2}$
}

\begin{abstract}
Velez, I. \& V.E. Thatcher: Five species of Lecithodendriidae (Trematoda) in bats from Colombia, including three new genera. Three new neotropical genera and species of Lecithodendriidae (Trematoda) are described from Colombian bats. Sturniratrema umbilicata gen. et sp. nov. from Stumira lilium differs from other genera in having an oval ventral depression, a dorso-ventrally curved cirrus and a genital pore lateral to the acetabulum.

Chiropterotrema antioquiense gen. et sp. nov. from Carollia perspicillata is distinguished by having the acetabulum within a triangular ventral pit as well as an separation between the the male and female genital pores.

Topsiturvitrema verticalia gen. et $s p$. nov. from Carollia perspicillata has a different orientation of the body, being nearly spherical but somewhat flatened in an anterior-posterior direction. This genus also has a deep ventral cleft separating two ventral lobes which are filled with eggs. Castroia silvai Travassos, 1928, is also reported from Anoura caudifer and Peropteryx macrotis. The first of these represents a new host record for the species. Addiotionally, Limatulum gastroides Macy, 1935, is reported from Lonchophyla robusta wich is another new host record.
\end{abstract}

\section{INTRODUCCION}

Lecithodendriidae es sin duda una de las familias mas comunes en tremátodos intestinales de murciélagos. Yamaguti, 1971, enumera 14 géneros y 30 especies en America neotropical, de los cuales cuatro géneros y cinco especies fueron encontradas en Colombia: Castroia silvai, Castroia amplicava y Limatulum gastroides por Martin, 1969; Prostodendrium calimaese, Proechimitrema phanerotermina y Oryzomytrema tristoma por Thatcher, 1982.

Los presentes tremátodos fueron colectados en murciélagos del departamento de Antióquia. Se capturaron 304 huéspedes de dos familias, 14 géneros y 25 especies. 10 especies resultaron parasitadas con tremátodos.

En el presente trabajo son descritos tres nuevos géneros y especies de Lecithodendriidae y también son reportados huéspedes nuevos para Castrois silvai Travassos, 1928, y para Limatulum gastroides, Macy, 1935.

1. Imelda Velez, Universidad de Antioquia, Departamento de Biología, Apartado Aéreo 1226, Medellín, Colombia.

2. Vernon E. Thatcher, INPA-DBA CP 478, 69011 Manaus, Brasil. 


\section{MATERIALES Y METODOS}

Los murciélagos fueron capturados con redes japonesas al atardecer y por la noche. Los animales vivos fueron transladados en bolsas de tela al laboratorio. A los individuos muertos se les extrageron los intestinos el mismo día y se fijaron en AFA. Los intestinos fueron lavados en agua corriente y luego fueron examinados para tremátodos con un estereo-binocular Wild. Los parásitos fueron coloreados con borax-carmin, deshidratados en alcohol $95 \%$ y fenol y aclarados con salicilato de metilo. Las placas permanentes se montaron en bálsamo del Canadá. Los dibujos fueron realizados con cámara lúcida y las medidas con un ocular micrométrico. Las medidas están dadas en micras $(\mu \mathrm{m})$ y los extremos estan seguidos del promedio entre paréntesis.

\section{SECCION SISTEMATICA}

\section{Familia: LECITHODENDRIIDAE}

Género: Sturniratrema gen. nov.

Diagnósis genérica: Lecithodendriidae. Cuerpo ovoide con una depresión central ovalada con bordes musculosos que comienzan posteriores a la ventosa oral y terminan al nivel posterior del acetábulo. Tegumento espinoso. Ventosa oral subterminal, bién desarrollada, mas grande que el acetábulo; prefaringe ausente, dorsal al acetábulo, esférica; esófago ausente; cecos cortos, preacetábulares, horizontales. Acetábulo bién desarrollado, en el fondo de la depresión central. Testículos ovoides, opuestos, postacetábulares. Saco del cirro elongado, dorsolateral al acetábulo con curvatura hacia éste, en forma de $\mathrm{U}$, contiene cirro y vesícula seminal. Poro genital lateral al acetábulo y marginaI a la cavidad ventral. Ovario un poco lobulado, al lado opuesto del saco del cirro. Vitelarios en pequeños folículos, dorsales, entre la ventosa oral y el acetábulo. Utero postacetabular, ocupando toda la región posterior. Vesícula excretora no observada; poro excretor terminal. Parásitos intestinales de Chiroptera.

Especie tipo: Sturniratrema umbilicata sp. nov.

Sturniratrema umbilicata sp. nov.

(Fig. 1)

Huésped: Sturnira lilium Geoffroy, 1810.

Lugar de infección: intestino delgado.

Procedencia: Puerto Triunfo, Departamento de Antioquia, Colombia.

Prevalencia: $1 / 30$.

Holótipo y dos parátipos: Harold W. Manter Laboratory, University of Nebraska, State Museum, Lincoln Nebraska; otros dos parátipos en la colección del Departamento dè Biología, Universidad de Antioquia, Colombia.

Etimologia: el nombre generido nuevo se refiere ao murciélago huésped y la designación específica se deriva de la depresión central ovalada característica del género.

Descripción (baseada en 10 especímenes medidos): cuerpo ovalado 450-680 (581) de largo por 360-460 (401) de ancho en la región ecuatorial. Tegumento espinoso hasta la región ecuatorial. Ventosa oral subterminal, 110-171 (128) de largo por 110200 (160) de ancho. Prefaringe ausente. Faringe esférica, 50-57 (54) de diámetro, dorsal la ventosa oral. Esófago ausente. Cecos cortos extendido lateralmente. Depresión 
Vol. 7(1-2), 1990

central 75-87 (84) de largo por 87-137 (116) de ancho; acetábulo 100-140 (118) de largo por 100-140 (128) de ancho. Testículos ovoides, laterales, post-acetábulares. Testículo izquierdo, 75-120 (96) de largo por 100-150 (116) de ancho. Testículo derecho, 62112 (85) de largo por 90-200 (126) de ancho. Saco del cirro lateral al acetábulo, 82-175 (133) de largo por 40-62 (60) de ancho, conteniendo cirro y vesícula seminal. Poro genital lateral al acetábulo en al lado opuesto al saco del cirro, posttesticular, $62-80$ (71) de largo por 80-120 (101) de ancho. Glándulas vitelinas preecuatoriales, laterales, entre la ventosa oral y el acetábulo, en pequeños folículos. Utero postacetabular parcialmente cubriendo la parte posterior de éstos. Huevos pequeños, $15-20 \times 7-10$. Vesícula excretora no observada; poro excretor terminal.

Sturniratrema gen. nov. se diferencia de los demás géneros de la familia en poseer una depresión ventral ovalada con bordes musculosos, en la cual esta localizado el acetábulo. El género nuevo también se distingue en tener un saco del cirro doblado dorsoventralmente y el poro genital al lado del acetábulo.

\section{Género: Chiropterotrema gen. nov.}

Diagnósis genérica: Lecithodendriidae. Cuerpo aplanado, ovoide, mas ancho que largo; tegumento espinoso. Ventosa oral subterminal, bién desarrollada, del mismo tamaño que al acetábulo; prefaringe ausente; faringe pequeña dorsal al acetábulo; esófago ausente; cecos corren en la margen anterior del cuerpo hasta el ecuador. Acetábulo bién desarrollado, preecuatorial, dentro de una pequeña cavidad triángular. Testículos ovoides, opuestos, postacetabulares. Saco del cirro grande, preecuatorial, sobre la parte anterior del testículo, contiene cirro y vesícula seminal; poro genital masculino lateral sobre el testículo. Ovario lobulado, al lado opuesto del saco del cirro, al margen del acetábulo. Vitelarios en pequeños folículos dorsales a la ventosa oral y los cecos. Utero postacetabular, ocupando toda la parte posterior del cuerpo; poro genital femenino separado del masculino. Vesícula excretora em forma de Y; poro excretor terminal. Parásitos intestinales de Chiroptera.

Especie tipo: Chiropterotrema antioquiense sp. nov.

\section{Chiropterotrema antioquiense sp. nov.}

(Fig. 3)

Huésped: Carollia perspicillata $\mathrm{L}$.

Lugar de infección: intestino delgado.

Procedencia: Puerto Triunfo, Departamento de Antióquia, Colombia.

Prevalencia: $2 / 93$.

Holótipo y dos parátipos: Harold W. Manter Laboratory, University of Nebraska, State Museum, Lincoln Nebraska; otros dos parátipos en la colección del Departamento de Biología, Universidad de Antioquia, Colombia.

Etimología: el nombre genérico nuevo se refiere al huésped Chiroptera; el nombre específico al Departamento de Antioquia donde se capturaron los huéspedes.

Descripción (basado en siete especímenes medidos): con los caractéres del género. Cuerpo 562-696 (629) de largo por 678-872 (775) de ancho. La ventosa oral subterminal, 111-152 (131) de largo por 135-164 (150) de ancho. Prefaringe ausente; faringe esférica; esófago ausente, bifurcación de los cecos inmediatamente después de la faringe, laterales hasta el ecuador. Acetábulo, 113-166 (140) de largo por 126-166 (146) de ancho. Testículo izquierdo 187-214 (200) de largo por 155-264 (210) de ancho. Testí- 
culo derecho 161-218 (221) de largo por 164-215 (190) de ancho. Saco del cirro 225 de largo por 117 de ancho; poros genitales laterales y separados, 12. Ovario 93-233 (163) de largo por 75-196 (136) de ancho. Glándula de Mehlis y ootipo no observados. Huevos numerosos y pequeños, 18-26 (22) X 8-13 (11). Vesícula excretora en forma de $Y$; poro terminal.

Chiropterotrema antioquiense sp. nov. se diferencia de los demas géneros de la familia em poseer el acetábulo dentro de una cavidad triangular que lo rodea, así como los poros genitales separados y laterales al acetábulo y el ovario lobulado.

Género: Topsiturvitrema gen. nov.

Diagnosis genérica:" cuerpo esférico, levemente aplanado en sentido anteroposterior y con una hendidura ventral que lo divide en dos hemisferios iguales. Tegumento liso. Ventosa oral subterminal, casi ecuatorial, bién desarrollada, mas grande que el acetábulo; prefaringe ausente; faringe esférica, dorsal al acetábulo; esófago ausente; cecos laterales hasta el ecuador. Acetábulo en el fondo de la hendidura posterior, posttesticular. Testículos ovoides, levemente lobulados, laterales, ecuatoriales. Saco del cirro al lado derecho del acetábulo. Poro genital en medio de la hendidura posterior. Ovario alargado, anterior a la ventosa oral. Vitelarios en pequeños folículos dorsales a la ventosa oral y los cecos. Utero en la parte dorsal y de los hemisferios. Vesícula excretora no observable; poro genital terminal. Parásitos intestinales de Chiroptera.

nal. Parásitos intestinales de Chiroptera.

Especie tipo: Topsiturvitrema verticalia sp. nov.

\section{Topsiturvitrema verticalia sp. nov.}

(Figs. 5-7)

\section{Huésped: Carollia perspicillata L.}

Lugar de infección: intestino delgado.

Procedencia: Puerto Triunfo, Departamento de Antióquia, Colombia.

Holótipo y dos parátipos: Harold W. Manter Laboratory, University of Nebraska, State Museum, Lincoln Nebraska; otros dos parátipos en la colección del Departamento de Biología, Universidad de Antioquia, Colombia.

Etimologia: el nombre genérico nuevo se refiere a la condición inversa de los órganos y la orientación invertida del cuerpo; proviene de la palabra en inglés "topsy-turvy" que significa "condición invertida" o "confusión y desorden".

Descripción (basada en cinco especímenes medidos): con los caractéres del género. Cuerpo redondo esférico, 300-350 (320) de largo por $290-430$ (392) de ancho en la región œntral. Tegumento liso. Ventosa oral grande, subterminal, 107-125 (121) de largo por 137-155 (149) de ancho; prefaringe ausente; faringe esférica, dorsal al acetábulo; esófago ausente; cecos cortos corriendo lateralmente hasta los testículos. Acetábulo, 68-87 (77) de largo por 87-112 (98) de ancho. Testículos levemente lobulados, laterales al acetábulo; testículo izquierdo, $62-87$ (72) de largo por $75-85$ (78) de ancho. Saco del cirro curvo, lateral al acetábulo, contiene cirro y vesícula seminal; poro genital en ia hendidura ventral. Ovario anterior a la ventosa oral, 100 de largo por 37 de ancho. Glándulas vitelinas en folículos en forma de media luna, paralelos a los cecos, dorsales a la ventosa oral. Utero ventral. Huevos grandes, 25-27 (26) X 9-12 (9). Vesícula excretora y poro excretor no observados. 
Topsiturvitrema verticalia sp. nov. se diferencia por la orientación del cuerpo que es totalmente diferente a todos los demás géneros de la familia Lecithodendriidae. Cuerpo esférico, levemente aplanado en sentido anteroposterior, con una hendidura ventral en cuyo fondo se encuentra el acetábulo; la ventosa oral central con el acetábulo casi posterior a ella.

En las Figs. 6 y 7 se observa la secuencia de los diferentes grados de madurez de la especie, donde es posible seguir el proceso de formación de la hendidura ventral y el desarrollo de los hemisferios. A medida que el tegumento va invaginandose la ventosa oral va desplazandose hacia la parte central del cuerpo.

\section{Castroia silvai Travassos, 1928}

(Fig. 4)

Huéspedes: Anoura caudifer Geoffroy, 1818 y Peropteryx macrotis Wagner, 1843. Lugar de infección: intestino delgado.

Procedencia: Puerto Triunfo, Departamento de Antióquia, Colombia.

Prevalencia: A. caudifer $1 / 1 ; P$. macrotis $1 / 8$.

El género Castroia, Travassos, 1928, se caracteriza por ser animales de cuerpo muy pequeño, en forma de media luna, o sea mas ancho que largo. Hasta ahora es un género endémico de Centro y Suramérica. Castroia silvai es aqui reportada por segunda vez en Colombia. Nuestros especímenes concuerdan en general con las descripciones anteriores (Travassos, 1928; Caballero, 1962; Martin, 1969), sólo se diferencian en que la presente es mas pequeña (281-342 X 542-803). Este es el primer registro del género Castroia en el murciélago Anoura caudifer (Phyllostomidae); hasta ahora siempre se ha encontrado en huéspedes del género Peropteryx (Emballonuridae) a escepción de Caballero, 1962, que lo encontró en Mycronycterys hirsuta (Phyllostomidae).

\section{Limatulum gastroides Macy, 1953}

(Fig. 2)

Huésped: Lonchophyla robusta Miller, 1912.

Lugar de infección: intestino delgado.

Procedencia: Puerto Triunfo, Departamento de Antioquia, Colombia.

Prevalencia: $1 / 8$.

Los presentes especímenes concuerdan con los descritos por Martin, 1969, en el sur de Colombia. El mismo autor y Duboes, 1964, ponen como sinónimos de Limatulum gastroides a Onchoterenatrema costarricensis, Caballero \& Brenes, 1957, Limatulum costarricensis, Caballero, 1964, y L. istmicus, Caballero, 1964, todos encontrados en el área neotropical. Lo llamativo de todos estos sinónimos es que los primeros especímenes descritos por Macy, 1935, son del norte de los Estados Unidos, Oregon, Wisconsin y Minnesota, aunque los huéspedes son del mismo género (Myotis) a los encontrados por Caballero, 1964, en Panamá, Nosostros consideramos que realmente son sinónimos, pués el ovario y el saco del cirro que se encuentran laterales al acetábulo varia mucho en la posición, lo cual puede prestarse para diferentes interpretaciones. El presente reporte es el primero para el huésped Lonchophyla robusta. 
Revta bras. Zool.

Fig. I.

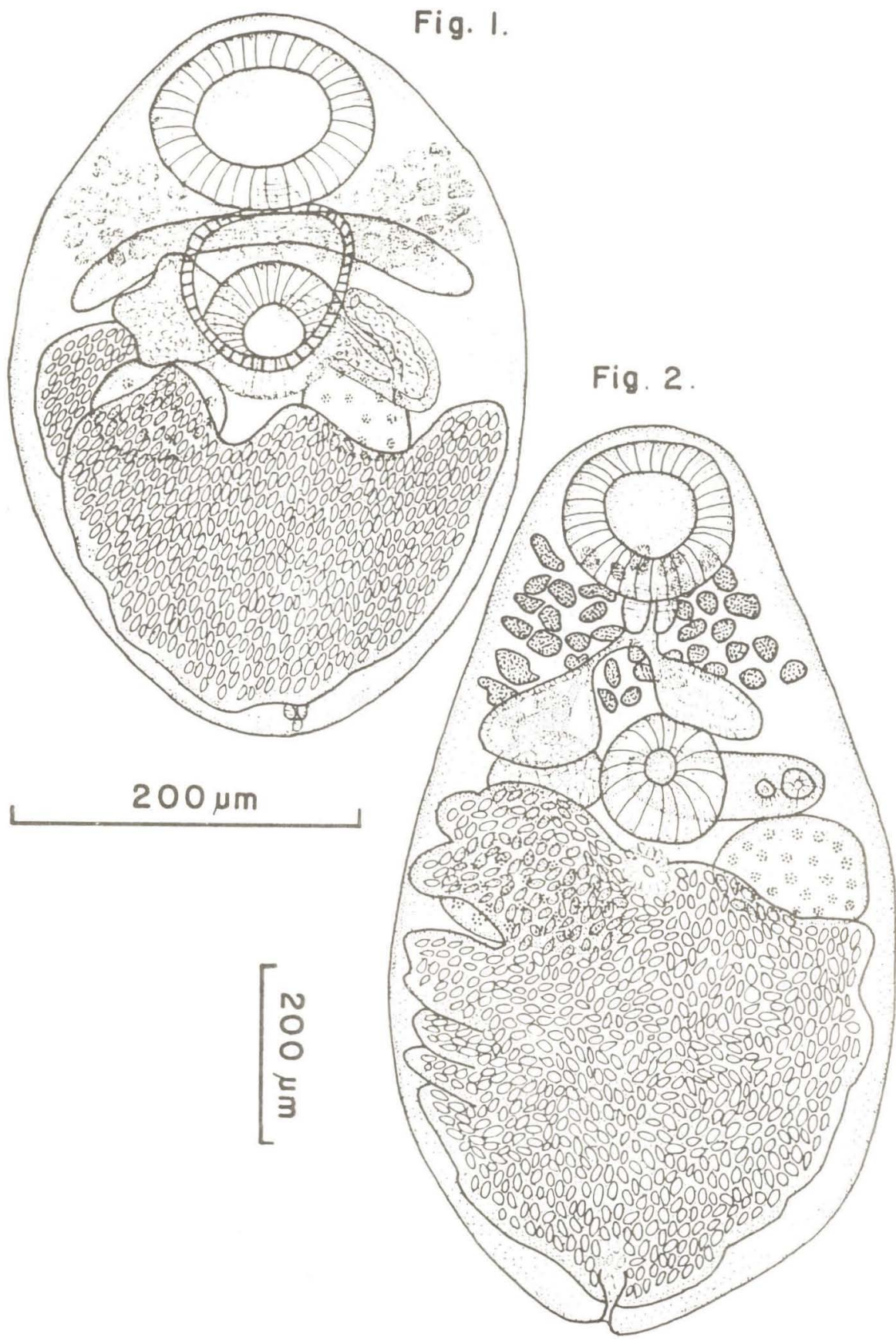

Fig. 1 - Sturniratrema umbilicata gen. et sp. nov. Vista ventral del holótipo.

Fig. 2 - Limatulum gastroides Macy, 1935. Vista ventral. 
Vol. 7(1-2), 1990

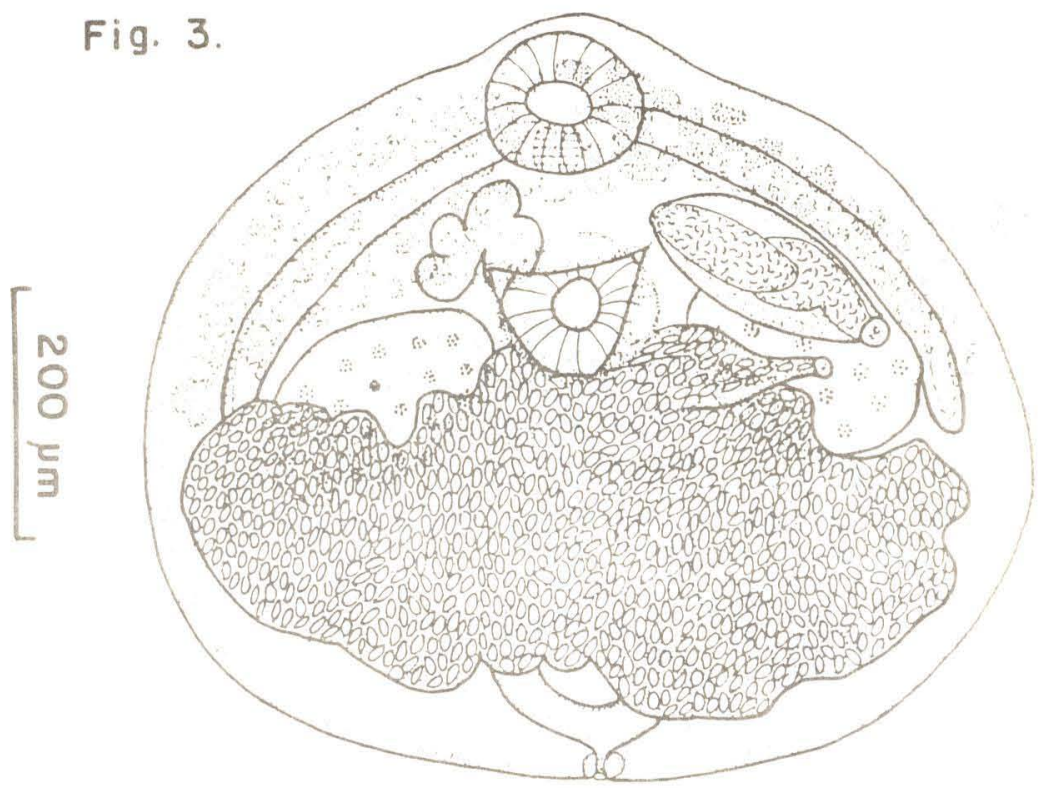

Fig. 4 .

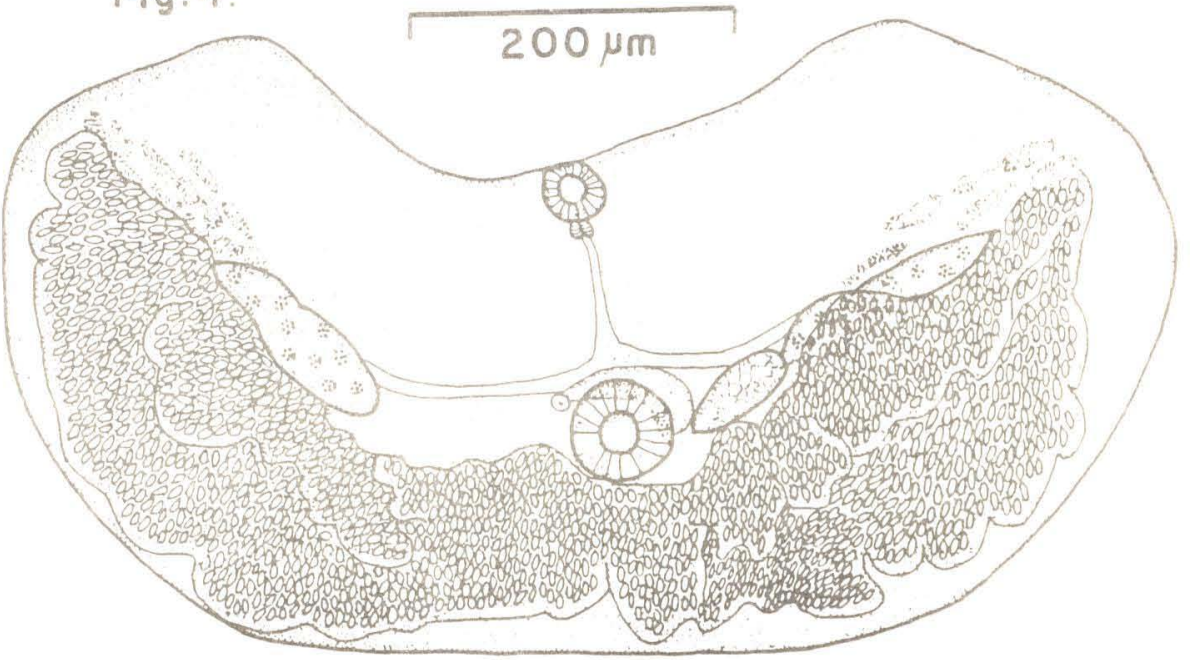

Fig. 3 - Chiropterotrema antioquiense gen. et sp. nov. Vista ventral del parátipo.

Fig. 4 -Castroia silvai Travassos, 1928. Vista ventral. 
Revta bras. Zool.

Fig. 5.

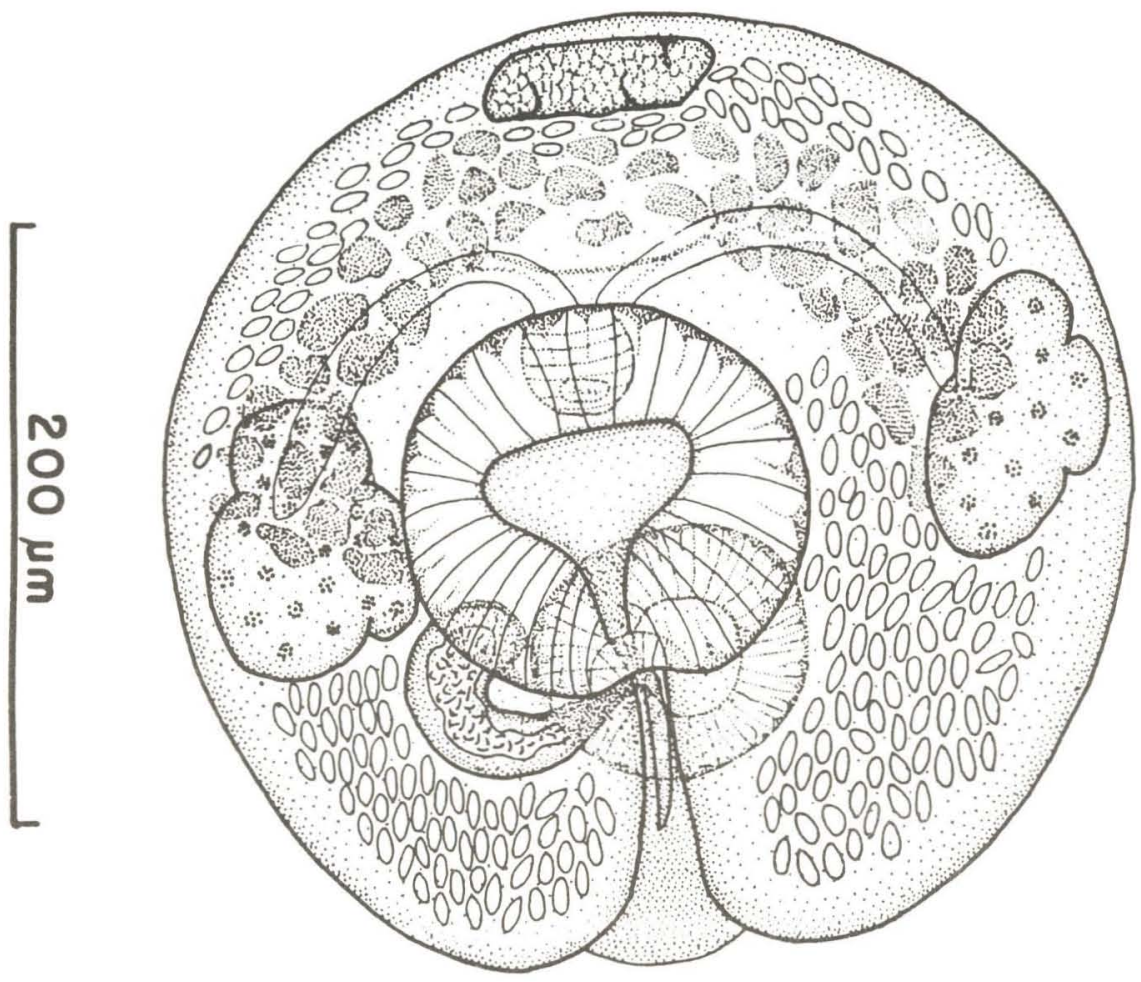

Fig. 5 - Topsiturvitrema verticalia gen. et sp. nov. Vista ventral del parátipo. 
Vol. 7(1-2), 1990

Fig. 6.

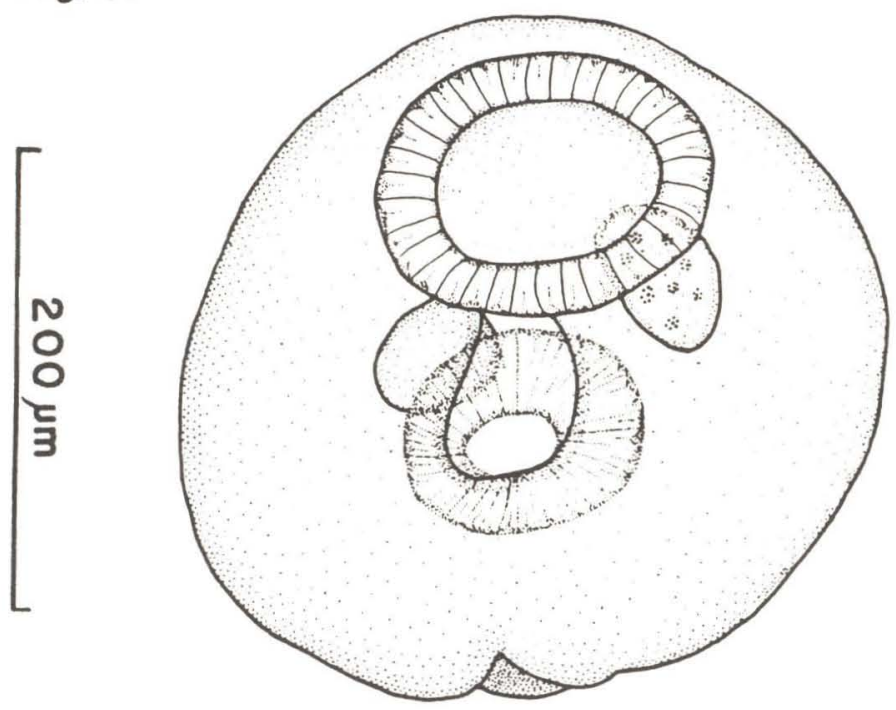

Fig. 7.

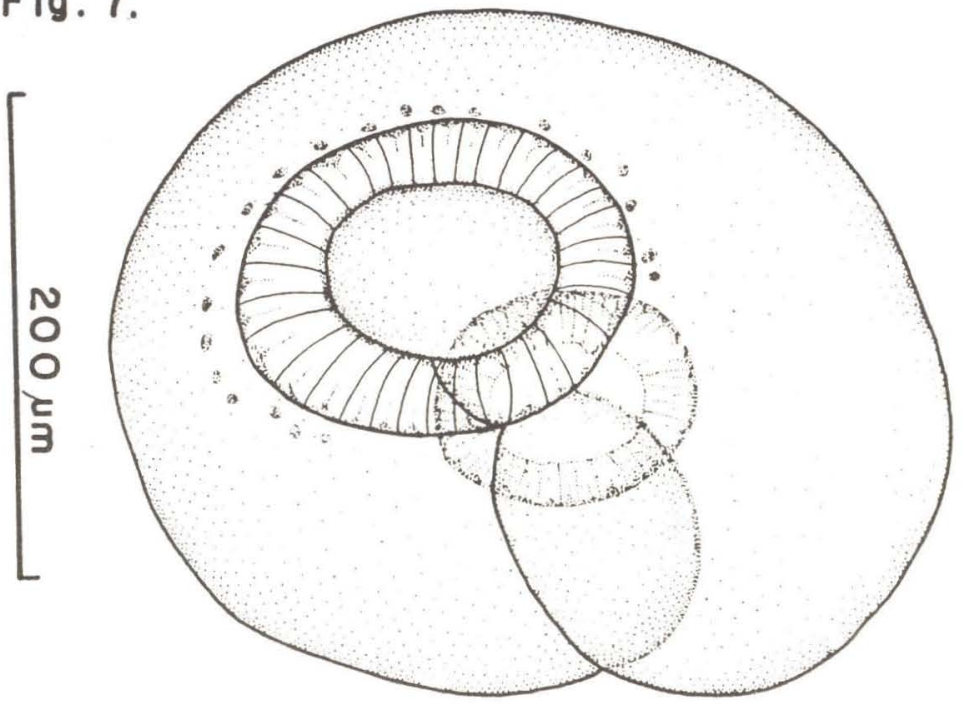

Figs. 6 y 7: Topsiturvitrema verticalia gen. et sp. nov. Secuencia de los estadios consecutivos de madurez de la especie. 


\section{AGRADECIMIENTOS}

Agradecemos al profesor Javier Muñoz, Departamento de Biología, Universidad de Antioquia, por la recolección y clasificación de los murciélagos y al tecnólogo Hugo Rios por la extracción de los intestinos en el campo. A el Centro de Investigaciones de la Facultad de Ciencias Exactas y Naturales de la U. de A. (CIEN) por el apoyo económico prestado para el presente estudio.

\section{REFERENCIAS}

CABALleRO y C., E., \& R.R. BRENES, M., 1957. Helmintos de la República de Costa Rica VI. Algúnos tremátodos de peces, reptiles y mamíferos. An Inst. Biol. Mexico 28: 217-240.

—. 1964b. Helmintos de la República de Panamá XXIV. Descripción de tres especies de tremátodos Lecithodendriidae que parasitan el murciélago Myotis nigricans (Schinz). An. Esc. Nac. Biol. 13: 73-82.

—- y F. Montero y G. 1962. Presencia de Castroia silvai Travassos, 1928, (Trematoda, Digenea) en Murciélagos de Costa Rica. An. Esc. Nac. Cienc. Biol. Mexico 12: 85-89.

DUBOIS, G., 1964. Contributions l'étude de trematodes de chiropteres. Les generes Limatulum Travassos, 1921, Limatuloides gen. nov. Suisse Zool. 71: 371-381.

MACY, R.M., 1935. A new trematode, Limatulum gastroides (Lecithodendriidae) from little brawn bat Myotis lucifugus. Proc. Helm. Soc. Wash. 2: 74-75.

MARTIN, D.R., 1969. Lecithodendriid trematodes from the bat Peropteryx kapleri in Colombia, including discussions of allometric growth and significance of ecological isolation. Proc. Helminth. Soc. Wash. 36 (2): 250-260.

THATCHER, V.E., 1982. Five neotropical species of Lecithodendriidae (Trematoda: Digenea) including three new genera, all from Panamian and Colombia mammals. Proc. Helminth. Soc. Wash. 49 (1): 45-55.

TRAVASSOS, L.P., 1928. Contribution á l'étude des Lecithodendriidae du Brasil. Mems. Inst. Oswaldo Cruz 21: 189-199.

YAMAGUTY, S., 1971. Synopsis of Digenetic Trematodes of Vertebrates, vols. I e II. Keigaku Publ., Co. Tokio, $1774 \mathrm{pp}$. 